\title{
Urodynamic Evaluation in Men Over 50 Years with Diabetes Mellitus and Lower Urinary Tract Symptoms - A Single Center Experience
}

\author{
Ragoori D', Sekar $\mathbf{H}^{2}$, Krishnamoorthy $\mathbf{S}^{2 *}$, \\ Kumaresan $\mathbf{N}^{2}$ and Ramanan $\mathbf{V}^{2}$ \\ ${ }^{1}$ Consultant Urologist, Asian Institute of Nephrology \& \\ Urology, India \\ ${ }^{2}$ Department of Urology, Sri Ramachandra Medical \\ College \& RI, India \\ *Correspondling author: Krishnamoorthy S, \\ Department of Urology, Sri Ramachandra Medical \\ College \& RI, Chennai, India
}

Received: May 20, 2016; Accepted: J anuary 24, 2017; Published: J anuary 26, 2017

\begin{abstract}
Diabetes Mellitus affects approximately 285 million people worldwide with a steady rise in incidence over the last decade. It has been projected that 438 million individuals would be affected with diabetes by the year 2030. In India it is estimated that presently 50.8 million individuals are affected by this disease, which is likely to go up to 87 million by the year 2030. The reasons for this escalation are due to various changes in lifestyle, longer life span than before (ageing) and low birth weight could lead to diabetes during adulthood. Benign Prostatic Hyperplasia (BPH) with Lower Urinary Tract Symptoms (LUTS) is a significant health problem affecting $50 \%$ of men older than 50 years of age and diabetes is another very common disease happening in the same age group.

Bladder involvement in Diabetes was recognized over 100 years ago and was established as a manifestation of diabetic neuropathy nearly 65 years ago. However it still remains an entity that is unappreciated and too frequently unrecognized. The exact incidence of voiding dysfunction caused by Diabetes Mellitus has not been clearly studied and is uncertain till date.
\end{abstract}

Keywords: Diabetes mellitus; Urinary tract symptoms

\section{Introduction}

Long standing diabetes can cause bladder dysfunction, involves autonomic neuropathy leading to functional sympathetic and possible sympathetic denervation of detrusor. Impaired detrusor function results in a lower maximum flow rate and an increase in post void residual urine [1,2]. Benign Prostatic Hyperplasia (BPH) can also cause lower urinary tract symptoms, including reduced maximum flow rate and increased post void residual urine $[3,4]$. However, the underlying pathology is different since $\mathrm{BPH}$ does not primarily impair detrusor function but rather enhances bladder outlet resistance via static and dynamic components. Since diabetes and $\mathrm{BPH}$ increase in prevalence with age, has to be expected that a major fraction of patients with $\mathrm{BPH}$ concomitantly suffer from diabetes and vice versa.

Most of the diabetic patients generally do not complain of bladder symptoms. However if specifically questioned, anywhere from 5-59\% of diabetics report symptoms of voiding dysfunction [5].

Diabetic cystopathy - the classical involvement or pattern of voiding dysfunction is a constellation of clinical and urodynamic findings associated with long term diabetes mellitus. The term diabetic cystopathy was coined by Frimodt-Moller in 1976 [6,8,9].Though the classical symptom of diabetic cystopathy has been well described these have not always been the pattern of voiding dysfunction in diabetes.

The patients usually have a varied symptomatic presentation. This may be due to the fact that most of these patients may have concomitant lesions such as benign prostatic hyperplasia, bladder or prostate cancer and infection. These conditions may mimic the symptoms of diabetic cystopathy and as a result diabetic patients complain of a variety of lower urinary tract symptoms.

The main differential diagnosis, at least in men is generally bladder outlet obstruction. Both these conditions produce a low flow rate and a similar symptomatic pattern. Only pressure / flow urodynamic study can differentiate between these two.

In developing countries like India where resources are limited, urodynamics is not widely available and hence not much work has been done to study the pattern of voiding dysfunction in diabetics. In a large outpatient practice in the department of urology at Sri Ramachandra Medical College and Research Institute, the clinicians are often confronted with long standing diabetic males, who present with lower urinary tract symptoms.

It poses a therapeutic challenge to the urologist treating, in deciding the line of management for these patients. It would be a difficult proposition unless and until the pattern of voiding dysfunction is clearly made out for which urodynamics remains the only answer.

Hence we embarked on this study to probe into the urodynamic evaluation of Indian diabetic men of age above 50 years with lower urinary tract symptoms. The main objectives of our study are (i) to determine the prevalence of bladder outlet obstruction and other urodynamic abnormalities in diabetic patients with LUTS and enlarged prostate; (ii) to assess the predictive value of non invasive tests for BOO diagnosis and (iii) to investigate the clinical significance of urodynamic studies in diabetic men.

\section{Materials and Methods}

Urodynamic study was performed in 35 male patients with
Austin J Urol - Volume 4 Issue 1 - 2017

ISSN: 2472-3606 | www.austinpublishinggroup.com

Krishnamoorthy et al. @ A All rights are reserved
Citation: Ragoori D, Sekar H, Krishnamoorthy S, Kumaresan N and Ramanan V. Urodynamic Evaluation in Men Over 50 Years with Diabetes Mellitus and Lower Urinary Tract Symptoms - A Single Center Experience. Austin J Urol. 2017; 4(1): 1052. 
Table 1: Age distribution of patients.

\begin{tabular}{|c|c|c|}
\hline Age (years) & No. of Patients & $\%$ \\
\hline $50-60$ & 8 & 22.85 \\
\hline $61-70$ & 11 & 31.42 \\
\hline $71-80$ & 11 & 31.42 \\
\hline $81-90$ & 5 & 14.28 \\
\hline
\end{tabular}

diabetes and voiding symptoms and the findings were analyzed. Data was collected in a pretested proforma meeting the objectives of the study.

The inclusion criteria are: age $>50 \mathrm{yrs}$, diabetes mellitus for a minimum duration of 5 years, IPSS score : between 7 to 30, Q.max $<15 \mathrm{ml} / \mathrm{sec}$ with a voided volume of atleast $125 \mathrm{ml}$ and patients with urinary retention.

The exclusion criteria are: Prior surgical intervention, carcinoma prostate, stricture urethra, neurological diseases, active infection and vesical calculus.

Hence patients who were long standing diabetics for a minimum of 5 years and lower urinary tract symptoms were selected for the study to find out the efficacy of urodynamic evaluation in deciding the treatment option for diabetic men with LUTS.

The parameters that were included in this present study were:

1. A detailed history of the type, duration and treatment for diabetes mellitus.

2. The voiding symptoms, their duration and IPSS score.

3. A careful physical examination.

4. A per rectal examination was done in all these patients by a single examiner to assess the prostate size, sphincter tone, perianal sensation and Bulbocavernous Reflex (BCR).

Ultrasound of abdomen was done to assess the status of the upper tracts, any other lesions in bladder (growth, diverticula or Calculi), prostate architecture, size and configuration (median lobe) and post void residual urine. Urine culture was done to rule out pyuria and UTI. In the presence of UTI, the patients were subjects to a course of antibiotic therapy and urine examination was repeated before performing the urodynamic evaluation. Patients were not subjected to UDE in the presence of an active infection.

Urodynamic evaluation consisted of multi-channel urodynamics measuring abdominal, vesical and detrusor pressures simultaneously. Following cystometrogram, pressure flow studies were done. Uroflometry was performed along with simultaneous recording of the vesical pressures. The machine used for this purpose was Urolab Janus IV (Life-Tech ${ }^{\bullet}$ Inc).

A $6 \mathrm{~F}$ and $10 \mathrm{~F}$ infant feeding tubes were introduced simultaneously into the bladder transurethrally. The bladder was then emptied completely using these tubes. The $10 \mathrm{~F}$ tube was used for bladder filling with saline at room temperature at a medium fill rate of $30-70 \mathrm{ml} /$ min. The vesical pressures were measured using the $6 \mathrm{~F}$ feeding tube. The intra abdominal pressures were recorded using a rectal tube with a water filled balloon. Both the pressures were then simultaneously recorded by the machine. Any involuntary vesical pressure rise that was associated with urgency was defined as Detrusor instability [7].

The bladder volume at which the patient complains of discomfort or develops a bladder contraction was defined as the bladder capacity. Once the bladder capacity was reached, the filling line (10F tube) was removed and the patient instructed to void and pressure flow recording were done.

Once the recordings were obtained, depending upon Bladder Outlet Obstructive Index (BOOI) and Bladder Contractility Index (BCI) the patients were grouped as follows:

$$
\text { BOOI }=\text { Pdet Qmax - 2Qmax }
$$$$
\mathrm{BCI}=\mathrm{Pdet} \mathrm{Qmax}+5 \mathrm{Qmax}
$$

Patients with BOOI $>40$ were classified as patients with bladder outlet obstruction. Patients who had BOOI of $>20$ and $<40$ were considered to be INDETERMINATE or EQUIVOCAL. Patients who had poorly sustained or weak detrusor contraction (BCI of less than 50) were considered to have detrusor underactivity.

Once the patients were grouped into their respective categories, their data was analyzed.

\section{Results}

The following are the observations made in this study. A total of 35 men aged 50 years and above with diabetes mellitus for a minimum duration of at least 5 years and lower urinary tract symptoms were analyzed.

Table 1 describes the age distribution of the patients in our study. The age of these patients ranged from 50 to 89 years with a mean age of 68.22 years. There were 11 patients $(31.42 \%)$ who presented in 7 th and 8 th decades of life and 5 patients (14.28\%) in the 9th decade of life.

There were 31 patients $(88.57 \%)$ with NIDDM and 4 patients (11.42\%) with IDDM with a mean duration of 11 years. 13 patients (37.14\%) were on insulin when they presented to us. The mean HbAlc of 35 patients was 7.48. There was no difference in the urodynamic findings when compared between diabetic men who were on oral hypogycelmic agents and those who are insulin dependent. Similarly, duration of diabetes mellitus had no implication on the eventual diagnosis.

All the patients included in the study had lower urinary tract symptoms with a mean duration of 17.6 months. They were categorized in to voiding LUTS and storage LUTS. Figure 1 describes the pattern of symptoms in our study.

All the 35 patients (100\%) had storage LUTS. None of them had voiding LUTS alone. Frequency of Micturition and Nocturia were the symptoms observed in all these patients. About 27 patients (77.14\%) had both voiding and storage LUTS. Eight patients (22.85\%) had storage LUTS alone. One patient presented with painful urinary retention.

Few patients in our study had peripheral neuropathy and diabetic retinopathy. 5 patients (14.28\%) had peripheral neuropathy and 6 patients $(17.14 \%)$ with diabetic retinopathy presented to us and when analyzed had no statistical significance between groups. 


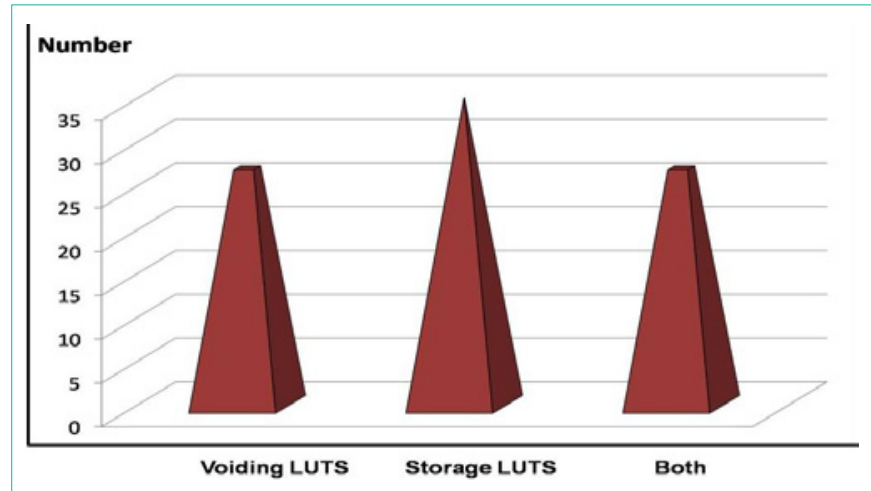

Figure 1: Pattern of symptoms.

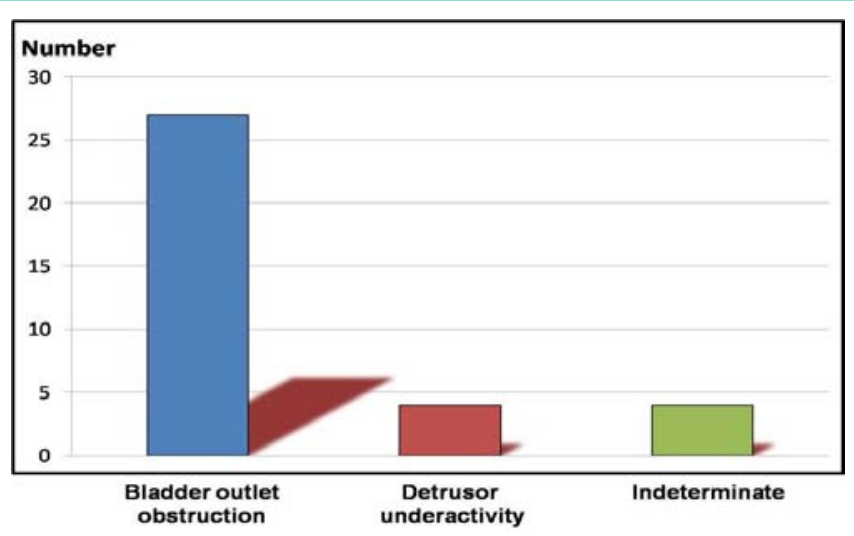

Figure 2: Summary of the urodynamic findings in our study.

Cystometric bladder capacity of these patients ranged from 150 $\mathrm{ml}$ to $1200 \mathrm{ml}$, the mean bladder capacity being $416 \mathrm{ml}$. Majority of patients $(\mathrm{n}=27)$ had bladder outlet obstruction $(77.14 \%)$, while 4 patients had detrusor underactivity $(11.42 \%)$ and another 4 patients had indeterminate findings (11.42\%). The classical finding of diabetic cystopathy i.e detrusor under activity was seen in 4 patients (11.42\%). Bladder outlet obstruction was present in a total of 27 patients (77.14\%) either as an isolated diagnosis or in combination with detrusor instability. Indeterminate findings on urodynamic evaluation were present in 4 patients (11.42\%). Figure 2 describes the summary of the urodynamic findings in all 35 patients in our study.

International prostate symptom score assessment was also done in all patients. The severity of the symptoms was assessed based on the IPSS score, where the mean score was 21.68. IPSS score in patients with bladder outlet obstruction was 22.66 and in patients with detrusor under activity it was 18 . There is a significant difference in the IPSS score between patients with BOO and DU. It gives a diagrammatic representation of the IPSS scores.

Hypertension and Chronic kidney disease were the other associated co morbid illnesses in our patients. About 23 patients (65.71\%) had associated hypertension and 6 patients (17.14\%) had chronic kidney disease.

Table 2 describes the details of those with hypertension and chronic kidney diseases. About 16 (69.56\%) out of the 23 hypertensive patients had BOO and 4 patients (17.39\%) had DU. There was no
Table 2: Co- Morbid illnesses in our study.

\begin{tabular}{|c|c|c|c|c|}
\hline Co-morbid & BOo & DU & Indeterminate & Total \\
\hline HTN & 16 & 4 & 3 & 23 \\
\hline CKD & 2 & 3 & 1 & 6 \\
\hline
\end{tabular}

statistical significance between the groups. However, 2 out of 27 patients with $\mathrm{BOO}$ had chronic kidney disease and 3 out of 4 patients with detrusor under activity had chronic kidney disease, which was statistically significant $(\mathrm{p}<0.0002)$.

The mean size of prostate measured by USG was $50.31 \mathrm{cc}$, with the smallest of $30 \mathrm{cc}$ and largest of $90 \mathrm{cc}$. The average size of prostate in patients with $\mathrm{BOO}$ was $53.33 \mathrm{cc}$ and in patients with DU it was $40.25 \mathrm{cc}$. There is a statistically significant difference in size of prostate between men with $\mathrm{BOO}$ and other groups.

Uroflowmetry was done in all the patients and the mean Q max was $9.94 \mathrm{ml} / \mathrm{sec}$. The mean Q max in patients with $\mathrm{BOO}$ was $10.5 \mathrm{ml} /$ $\mathrm{sec}$ whereas in patients with DU the Q max was $9.4 \mathrm{ml} / \mathrm{sec}$. The overall mean post void residual urine was $69.11 \mathrm{ml}$. There was no statistical difference in maximum urinary flow rate between different groups.

The final analysis of the urodynamic evaluation data of 35 patients has shown the following results.

The overall cystometric bladder capacity of these patients ranged from $150 \mathrm{ml}$ to $1200 \mathrm{ml}$, with the mean bladder capacity being 416 $\mathrm{ml}$. About 27 patients had bladder outlet obstruction (77.14\%), with 4 patients having detrusor underactivity $(11.42 \%)$ and another 4 patients having indeterminate findings (11.42\%)

The classical finding of diabetic cystopathy i.e detrusor under activity was seen in 4 patients (11.42\%). However there was one patient with impairment of detrusor contractility. Bladder outlet obstruction was present in a total of 27 patients (77.14\%) either as an isolated diagnosis or in combination with detrusor instability. Indeterminate findings on urodynamic evaluation were present in 4 patients $(11.42 \%)$.

\section{Discussion}

Frimodt Moller (1976) did an extensive work in voiding dysfunction in diabetics and coined the term Diabetic Cystopathy to describe the involvement of lower urinary tract by this disease [6-9].

Lower Urinary Tract Symptoms (LUTS) increase with age and moderate to severe LUTS occur in approximately $25 \%$ of men over 50 years of age. The pathophysiology of LUTS is multifactorial. Bladder Outlet Obstruction (BOO) is one of the main causes of LUTS; but detrusor factors such as detrusor instability and impaired contractility can contribute to the development of LUTS. Diabetes mellitus also affects bladder function producing LUTS; characterizing diabetic cystopathy is also common in BPH. Similarly, urgency and frequency are associated with both DM induced detrusor instability and $\mathrm{BPH}$. The distinction between LUTS secondary to DM and LUTS secondary to BPH is difficult to disentangle, and often LUTS secondary to DM overlaps LUTS secondary to BPH and vice versa. It is in such a situation where urodynamic evaluation plays an important role to distinguish the primary cause of LUTS and thereby helping the urologist in deciding the appropriate line of management (Figure 2). In addition to this, some authors acknowledge that urodynamic 
analysis can be used to predict the symptomatic outcome after TURP.

Analysis of the urodynamic data in our study showed that the classical finding of Diabetic cystopathy viz. detrusor under activity was not the most predominant form of voiding dysfunction.

One of the very first studies on Diabetes population by Frimodt Moller showed that there was an incidence of $38 \%$ of diabetic cystopathy \& $26 \%$ had Bladder outlet obstruction [6,9].

However the subsequent studies showed that diabetic bladders have a varied presentation.

Studies by Kitami et al as 173 diabetic patients (male 78 / female 95) showed than in addition to classical findings as increased volume at the first desire to void and decreased maximum vesical pressure, diabetic patients showed varieties of dysfunction such as overactive bladder (14.5\%), low compliance bladder (11.0\%) and loss of detrusor-external sphincter dyssynergia (31.7\%) [10].

In a subsequent study by Starer and Libow, they showed that in 23 diabetic patients with urinary incontinence, detrusor instability was found in $61 \%$ and $9 \%$ had detrusor areflexia $[11,12]$. They also observed that in their series all the patients presenting with retention had detrusor under activity.

Likewise Ho et al [13-15] analyzed the urodynamic findings of 94 diabetic patients with a variety of lower urinary tract symptoms. Over active bladder was seen in $36.2 \%$ of diabetics with a higher percentage of increased bladder sensation and detrusor overactivity, lower peak flow rate, greater post void residue volume, and lower bladder voiding efficiency. On pressure flow studies, the over active bladder group had a higher percentage of bladder outlet obstruction (26.5 vs. 6.7\%). Since they had excluded women with other causes of bladder outlet obstruction such as anti-incontinence surgery or pelvic organ prolapse, [16] it is likely that the majority of bladder outlet obstruction in this group of patients was due to functional obstruction (sometimes termed sphincter dyssynergia. DCP begins with selective damage to autonomic afferent nerves, leaving motor function intact but impairing the sensation of bladder fullness [17-20].

Kaplan and Blaivas [9] in a series of 182 patients showed that 55\% had detrusor hyperreflexia, $23 \%$ had impaired detrusor contractility $\& 19 \%$ has detrusor areflexia. They also had an incidence of $36 \%$ of bladder outlet obstruction.

Chancellor and Blaivas in a series of 43 patients observed that $33 \%$ had Detrusor over activity \& $23 \%$ had Detrusor under activity [12].

A study by Lee et al [16] compared the voiding behavior of 194 women with type 2 diabetes with that in 162 control women, using a lower urinary tract symptoms questionnaire and uroflowmetry. They found that diabetic women had significantly higher nocturia scores, weaker urinary streams, reduced voided volumes, and lower maximal flow rates. Residual urine volume $(100 \mathrm{ml}$ or greater) occurred in a significantly higher proportion of diabetic subjects (13.9 vs. $1.8 \%$ of controls).

Our study also proved that bladder under activity was not the major presentation in male patients with voiding dysfunction. In our series only $11.42 \%$ of the patients had detrusor under activity. (Table
Table 3: Showing the comparison of incidence of detrusor underactivity in various studies.

\begin{tabular}{|c|c|}
\hline \multicolumn{2}{|c|}{ COMPARISION OF THE INCIDENCE OF DETRUSOR UNDER ACTIVITY } \\
\hline Frimodt Moller & $38 \%$ \\
\hline Kaplan and Blaivas et al & $19 \%$ \\
\hline Chancellor and Blaivas et al & $23 \%$ \\
\hline Starer and Libow & $9 \%$ \\
\hline Our Study & $\mathbf{1 1 . 4 2 \%}$ \\
\hline
\end{tabular}

Table 4: Showing the comparison of incidence of bladder outlet obstruction in various studies.

\begin{tabular}{|c|c|}
\hline \multicolumn{2}{|c|}{ COMPARISON OF THE INCIDENCE OF BLADDER OUTLET } \\
\hline OBSTRUCTION & $26 \%$ \\
\hline Frimodt Moller & $56 \%$ \\
\hline Kaplan \& Blaivas & $\mathbf{7 7 . 1 4 \%}$ \\
\hline Our Study & \\
\hline
\end{tabular}

3) describes the comparison of the incidence of detrusor underactivity amongst various studies.

Of the 35 patients who were analyzed with urodynamic study, bladder outlet obstruction was diagnosed in 27 patients (77.14\%).

Table 4 describes the comparison of the incidence of bladder outlet obstruction amongst various studies.

Four patients in our study presented with indeterminate findings -where the voiding pressures were normal but the patients had a poor flow. It was difficult to discern whether it was an early stage of diabetic cystopathy. Hence it was planned to put these patients on periodic follow up and repeat urodynamic study later.

Most of our patients had presented to us with symptom of nocturia and were labeled to have the symptom due to diuresis which is an inherent part of diabetes. Urodynamic helped us to find that a majority of these patients in fact had an overactive bladder.

Thus our study elicited a good overview for clinicians who treat these patients. It helped us in differentiating patients with under activity and patients with bladder outlet obstruction, so that appropriate therapy can be instituted.

\section{Conclusion}

Diabetic patients with voiding dysfunction have varied urodynamic findings. Classical diabetic cystopathy is not the most common cause of voiding dysfunction in a significant percent of male diabetics. Bladder outlet obstruction occurs very commonly in diabetic patients presenting with obstructive symptoms. Detrusor under activity is predominantly seen in patients with diabetes mellitus with LUTS and associated chronic kidney disease. Urodynamic study plays an important role in helping the urologist to decide the line of management in diabetic male patients with LUTS.

\section{References}

1. Williams textbook of Endocrinology (12 $2^{\text {th }}$ ed.). Philadelphia: Elsevier/ Saunders. 978-1-4377-0324-5.

2. Shaw JE, Sicree RA, Zimmet PZ. Global estimates of the prevalence of diabetes for 2010 and 2030. Diabetes Res Clin Pract. 2010; 87: 4-14. 
3. Pradeepa R, Deepa R, Mohan V. Epidemiology of diabetes in India-current perspective and future projections. J Indian Med Assoc. 2002; 100: 144-148.

4. Jordan WR, Crabtree $\mathrm{JH}$. Paralysis of the bladder in diabetic patients. Arch Intern Med. 1935; 55: 17-25.

5. Wein AJ. Pathophysiology and categorization of voiding dysfunction, In Campbell's Urology, $8^{\text {th }}$ edition. 2002; 956-957.

6. Frimodt-Moller C. Diabetic cystopathy: A review of the urodynamic and clinical features of neurogenic bladder dysfunction in diabetes mellitus. Dan Med Bull. 1978; 25: 49-56.

7. Nitti VW. Cystometry and abdominal pressure monitoring. Practical Uodynamics. Philadelphia, WB Saunders. 1998; 38-51.

8. Walsh PC, Wein AJ, Retik AB, Vaughan ED. Neuromuscular dysfunction of the lower urinary tract and its treatment. Campbell's Urology. 1998; 1: 967968.

9. Kaplan SA, Alexise TE, Jerry G. Blaivas Urodynamic findings in patients with diabetic cystopathy. J Urol. 1995; 153: 342-344.

10. Kitami K. Vesicourethral dysfunction of diabetic patients. Nippon Hinyokika Gakkai Zasshi. 82; 1074-1083.

11. Starer P, Libow L. Cystometric evaluation of bladder dysfunction in elderly diabetic patients. Arch Intern Med. 1990; 150: 810-813.

12. Chancellor MB, Blaivas JG. Diabetic neurogenic bladder: Practical Neurourology, Boston, Butterorth, Heinemann. 1995; 149-154.
13. $\mathrm{Ho} \mathrm{CH}$, Tai $\mathrm{HC}, \mathrm{Yu} \mathrm{HJ}$. Urodynamic findings in female diabetic patients with and without overactive bladder symptoms. Neurourol Urodyn. 2010; 29: 424-427.

14. Ho MH, Bhatia NN, Khorram O. Physiologic role of nitric oxide and nitric oxide synthase in female lower urinary tract. Curr Opin Obstet Gynecol. 2004; 16: 423-429.

15. Ho MH, Yip S, Bhatia NN. Lower urinary tract dysfunctions in women with diabetes mellitus. Curr Opin Obstet Gynecol. 2007; 19: 469-473.

16. Lee WC, Wu HP, Tai TY, Liu SP, Chen J, Yu HJ. Effects of diabetes on female voiding behavior. J Urol. 2004; 172: 989-992.

17. Charles M. Clark Lee DA. Prevention and treatment of the complications of diabetes mellitus. N Eng J Med. 1995; 332: 1210-1216.

18. Tong, Ching $Y$, Chin. Role of sorbitol in the upregulation of urinary bladder M2 muscarinic receptors in streptozotocin-induced diabetic rats. Neurourolurodyn. 2002; 21: 154-159.

19. King $\mathrm{RH}$. The role of glycation in the pathogenesis of diabetic polyneuropathy. Molpathol. 2001; 54: 400-408.

20. Sabin T. Classification of peripheral neuropathy: The long and short of it. Muscle Nerve. 1986; 9: 711-719.
Austin J Urol - Volume 4 Issue 1 - 2017

ISSN: 2472-3606 | www.austinpublishinggroup.com

Krishnamoorthy et al. (C) All rights are reserved
Citation: Ragoori D, Sekar H, Krishnamoorthy S, Kumaresan N and Ramanan V. Urodynamic Evaluation in Men Over 50 Years with Diabetes Mellitus and Lower Urinary Tract Symptoms - A Single Center Experience. Austin J Urol. 2017; 4(1): 1052 